[30-39 yrs], 17\% [40-49 yrs], 19\% [50-59 yrs] and 23\% [older than $60 \mathrm{yrs}$ ].

Conclusion Falls down stairs or escalators is relatively common, in addition to a large percentage of people having a fear or feeling uncomfortable.

Learning Outcomes Crowd control at events should include supervisors near stairs or escalators.

\section{P4.013 EMPOWERING THE NEXT GENERATION OF SAFETY AMBASSADORS - NATIONAL SAFETY SCIENCE CAMPAIGN}

Naina Urs*, Lakshmi Nair*. Underwriters Laboratories, Bengaluru, India

10.1136/injuryprev-2021-safety.216

Context Aligned to SDG 4.7, the National Safety Science Campaign(NSSC) by Underwriters Laboratories in India aims to create a culture of safety in young minds and empower India's next generation of safety ambassadors.

Core initiatives

a. National Safety Science Quiz for grades 8 to 10 is aimed at imparting safety science knowledge

b. Poster Contest for grades 4 to 7 gives a platform to creatively unleash their thoughts on safety

c. Safety Impact films for ages 16 to 25 brings highlights the brilliant safety innovations being built by young students

d. Safer Schools Safer India is for primary level to help them learn about fire safety by following the story of our in-house comic character 'Bittoo'.

e. Safety clubs for middle schools is based on experiential learning for students to understand the science behind safety.

Outcomes Reached close to 57 million people across the country through this campaign.

Over 77,000 children from 2000 schools registered for the competitions in 2019. Safety Impact Films, piloted in 2019, garnered over 200 entries of which the top 5 innovations were chosen. Safety club program, piloted in 2019, saw a participation of 20 schools including 135 teachers and over 2000 students. With the Safer Schools Safer India program we trained 467 teachers and through them reached out to 26,879 students.

Learnings

- Need to influence the key stakeholders for safer school ecosystem, build awareness and promote the concept among children

- A combination of art and science to integrate safety into the curriculum.

\section{P4.014 A NOVEL ADOLESCENT ROAD TRAFFIC INJURY PREVENTION ENGAGEMENT PROJECT}

${ }^{1}$ Jessica Landry*, ${ }^{2}$ Nimsy Carolina Ramos Velásquez, ${ }^{2}$ María Isabel Bolaños Reyna, ${ }^{1}$ Cinnamon Dixon. 'University of Colorado/Children's Hospital Colorado, Aurora, USA; ${ }^{2}$ Trifinio Center for Human Development, Guatemala

10.1136/injuryprev-2021-safety.217

Background Injury accounts for 30\% of adolescent deaths in Guatemala; more than one-third are due to road traffic injuries (RTI). Little has been documented about adolescents who are most affected by RTI. This Photovoice project aimed to engage a cohort of rural Guatemalan adolescents to assess and voice perceived RTI risk factors and community strengths.

Methods The project enrolled 10 adolescents participating in a local Youth Leadership program. In-classroom group discussions were held with participants to identify RTI perceived risk and protective factors; adolescents then conducted Photovoice (photo-exploration) of their community's injury risks/ strengths.

Results RTI Risks: Five risk factors were identified during inclass discussion: desire for adrenaline, high speed travel, lack of traffic lights, distracted driving, and animals in streets. Adolescent photo-documentation identified an additional eleven risk factors: lack of separation between pedestrians and traffic, motorcycle-taxis, lack of curbs, steep drop-offs from the streets, narrow bridges, multiple passengers on motorcycles and in truck beds, young drivers, young children on motorcycles, broken glass and lack of helmets.

Community Strengths: Two community strengths were identified during classroom discussion: fences around homes and speed bumps. Photo-documentation resulted in identification of an additional six community strengths: guardrails, helmets, protective clothing, speedbumps in front of schools, school fences and security guards.

Conclusion RTI is a significant cause of adolescent mortality worldwide. Interactive injury prevention activities including Photovoice are important tools for motivating and involving this at-risk population to improve their understanding of injury risk and protective factors, which could result in increased safe behaviors.

\section{P4.015 COMMUNITY PREVENTION OF PROBLEMATIC CONSUMPTION 'FEEL GOOD, ENJOY WITHOUT EXCESSES'}

Nadia Rivera Leinecker*. Faculty of humanities and social sciences. Jujuy National University, El carmen, argentina

10.1136/injuryprev-2021-safety.218

In Jujuy province, alcohol consumption and acute poisoning are related to massive recreational events. Reducing risks and damage in this context is important.

The 'FEEL GOOD, ENJOY WITHOUT EXCESSES' program is a public policy which implements preventive actions articulated with other government departments and society. This program develops processes of care and protection inside recreation spaces, based on two lines: 1) Establish guidelines for responsible consumption and self-care. 2) Reduce risks and damage in nighttime contexts.

Strategy: Environmental prevention, advertising of care guidelines, staff training to detect risk; dissemination of protocol of poisoning or health emergency and creation of the health police.

Application

- Advertising of care and prevention guidelines.

- Presence of preventive agents for early risk detection.

- Stuff training (bartenders, cleaners, etc).

- Hydration and recovery friendly spaces creation.

- Activation of protocol in intoxication or emergency situations.

- Integrity and security protection in charge of the health police.

Results Applied in massive events between the years 2017 and 2019: Carnival (60 hours and 125,000 people) and Student Parties (84 hours and 235,000 people) it was observed: 